\title{
Ultra-efficient frequency comb generation in AlGaAs-on-insulator microresonators
}

\author{
Lin Chang, ${ }^{1,+}$ Weiqiang Xie,,$^{1,+,}$ Haowen Shu, ${ }^{1,2,+}$ Qi-Fan Yang, ${ }^{3}$ Boqiang Shen, ${ }^{3}$ Andreas Boes, ${ }^{1,4}$ Jon D. Peters, ${ }^{1}$ Warren Jin, ${ }^{1}$ Chao Xiang, ${ }^{1}$ \\ Songtao Liu, ${ }^{1}$ Gregory Moille, ${ }^{5}$ Su-Peng Yu, ${ }^{6}$ Xingjun Wang, ${ }^{2}$ Kartik Srinivasan, ${ }^{5}$ Scott B. Papp, ${ }^{6}$ Kerry Vahala, ${ }^{3}$ and John E. Bowers ${ }^{1}$ \\ ${ }^{1}$ Department of Electrical and Computer Engineering, University of California, Santa Barbara, CA 93106, USA \\ ${ }^{2}$ State Key Laboratory of Advanced Optical Communications System and Networks, Peking University, Beijing, 100871, China \\ ${ }^{3}$ T. J. Watson Laboratory of Applied Physics, California Institute of Technology, Pasadena, California 91125, USA \\ ${ }^{4}$ School of Engineering, RMIT University, Melbourne, VIC 3000, Australia \\ ${ }^{5}$ Microsystems and Nanotechnology Division, National Institute of Standards and Technology, Gaithersburg, MD 20899, USA \\ ${ }^{6}$ Time and Frequency Division, National Institute of Standards and Technology, Boulder, CO 80305 USA \\ weiqiangxie@ucsb.edu \\ $\uparrow$ All three authors contributed equally to this work
}

\begin{abstract}
We demonstrated ultra-efficient frequency comb generation in AlGaAs-on-insulator ring resonators that have a quality factor beyond $1.5 \times 10^{6}$. The threshold power is as low as 36 W. (C) 2020 The Author(s) OCIS codes: (190.4390) Nonlinear optics, integrated optics; (190.4380) Nonlinear optics, four-wave mixing; (160.6000) Semiconductor materials.
\end{abstract}

The extensive research on integrated nonlinear photonics in the last few years has opened up many new opportunities for on-chip integrated photonics, ranging from spectroscopy to atomic clock applications [1], [2]. The demand to construct efficient nonlinear devices has motivated the development of different material platforms in nonlinear photonics. So far, the dominating nonlinear materials used in integrated photonics are usually based on dielectrics, such as silica, $\mathrm{Si}_{3} \mathrm{~N}_{4}$ and so on. Recently, the significant reduction of loss in those platforms has enabled comb generation threshold power in the sub-milliwatt region. However, it is very challenging to integrate those devices into advanced photonic integrated circuits (PICs) along with other photonics components, such as lasers, amplifiers, modulators and detectors, as the dielectric material platform is not compatible with active devices. Another drawback for those dielectric microresonators is that they require extremely high quality $(Q)$ factors $(>10$ million) for efficient nonlinear operation, which poses significant challenges in the fabrication process.

The newly developed nonlinear platform, $(\mathrm{Al}) \mathrm{GaAs}$ on insulator, provides an attractive solution to overcome these problems [3]. (Al)GaAs has one of the highest third order nonlinear coefficient $\left(n_{2}=2.6 \times 10^{-13} \mathrm{~cm}^{2} \mathrm{~W}^{-1}\right)$, orders of magnitude higher compared to those of commonly used nonlinear materials, which greatly relieves the requirements for high $Q$ factors in microresonator fabrication [4]. Furthermore, $(\mathrm{Al}) \mathrm{GaAs}$ and its alloys have been widely used as an active medium in the PIC industry. Hence, such a platform is compatible for the integration of active and nonlinear optical elements, which is essential for future nonlinear photonic applications.

Here, we present ultra-high efficiency frequency comb generation using AlGaAs on insulator microresonators with unprecedented high $Q$ factors (beyond 1.5 million). The waveguides are fully etched with sub-micron dimensions and exhibit anomalous dispersion at $\mathrm{C}$ band. For a $1 \mathrm{THz}$ comb, the threshold power is only $\sim 36 \mathrm{~W}, 100$ times lower relative to previous semiconductor resonators and 10 times lower compared to the state-of-the-art results from integrated dielectric micro-resonators. With $300 \mathrm{~W}$ pump power we were able to generate a frequency comb that covers a spectral range of $\sim 250 \mathrm{~nm}$.

(a)

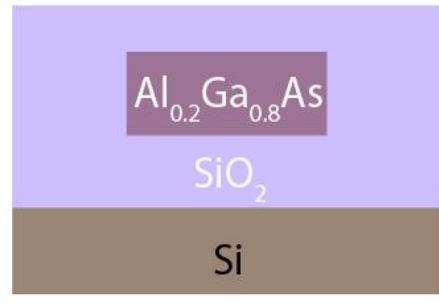

(b)

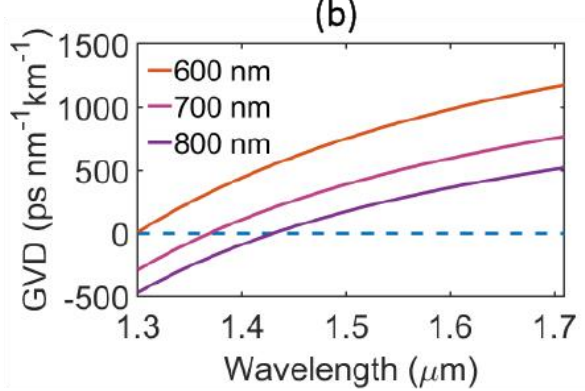

(c)

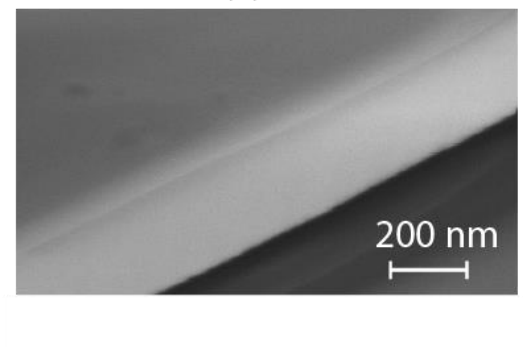

Fig.1. (a) Schematic drawing of the AlGaAsOI waveguide cross section; (b) simulated GVD of $400 \mathrm{~nm}$ thick AlGaAsOI waveguides with different widths; (c) SEM image of the sidewall of the waveguide.

One advantage of $\mathrm{Al}_{\mathrm{x}} \mathrm{Ga}_{1-\mathrm{x}} \mathrm{As}$ for nonlinear optical applications is that a higher $\mathrm{Al}$ compound increases the material's bandgap, which is essential to avoid two photon absorption, especially at C-band telecom wavelengths. In 
this work we used an $\mathrm{Al}$ concentration of $x=0.2$, which corresponds to a bandgap of $743 \mathrm{~nm}$. Another requirement for comb generation in microresonator is to use a waveguide with anomalous dispersion at the pump wavelength. In our case we chose the thickness of the AlGaAs layer to be $400 \mathrm{~nm}$. The simulated dispersions of waveguides with different width are plotted in Fig. 1(b). The mode size is 4 times smaller compared to that of common $\mathrm{Si}_{3} \mathrm{~N}_{4}$ waveguides for comb generation, thanks to the stronger refractive index contrast. The smaller mode size greatly enhances the photon density and thus improves the efficiency of the nonlinear optical process.

The fabrication for the $\mathrm{Al}_{0.2} \mathrm{Ga}_{0.8} \mathrm{As}$ devices is similar to the process described for the fabrication of the GaAs on insulator platform [5]. Here, we further optimized the lithography and etching process [6], which leads to smoother waveguide sidewalls, as shown in Fig. 1(c).

Fig. 2(a) shows the transmitted spectrum of a resonance of a ring resonator with $1 \mathrm{THz}$ FSR. The intrinsic quality factor of this resonator is extracted to be $1.5 \times 10^{6}$, which corresponds to a propagation loss around $0.4 \mathrm{~dB} / \mathrm{cm}$. For the first time, a III-V semiconductor nanowire waveguide suitable for nonlinear optics exhibits a waveguide loss that is as low as those of silicon and even comparable with many other dielectric waveguides.

(a)

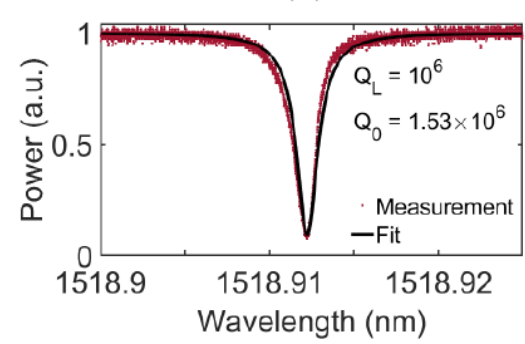

(b)

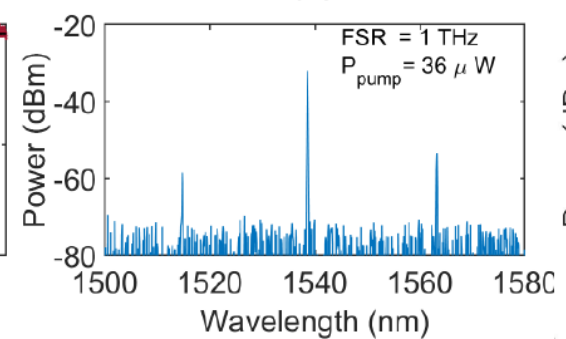

(c)

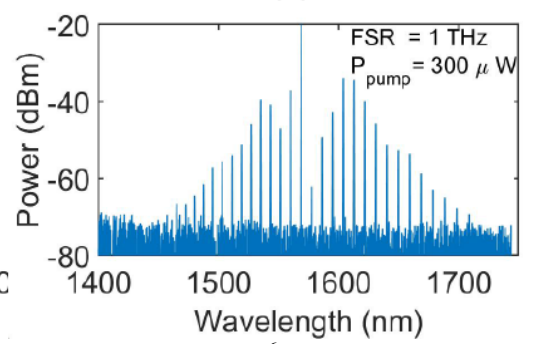

Fig.2. (a) Measured transmission spectrum of resonance. The intrinsic quality factor is $1.5 \times 10^{6}$, calculated by fitting a Lorentzian Function; Frequency comb spectrum from a $1 \mathrm{THz}$ resonator under pump power of (b) 36 W and (c) $300 \mathrm{~W}$

Such high $Q$ enabled a record low threshold power of $\sim 36$ W for comb generation, two order of magnitude lower compared to the previous results of semiconductor resonators as shown in Fig. 2(b). When increasing the pump power to $300 \mathrm{~W}$, the span of the comb extended over $250 \mathrm{~nm}$ range (Fig. 2(c)). Such pump power is still below the threshold power of the state of the art integrated dielectric microresonators.

In conclusion, we demonstrated a low loss $\mathrm{AlGaAsOI}$ platform and applied it to create microcavities with a $Q$ factor beyond $1.5 \times 10^{6}$. These high-Q devices in combination with the high nonlinearity of the AlGaAsOI waveguides enabled ultra-efficient frequency combs. A record low threshold power around $36 \mathrm{~W}$ for $1 \mathrm{THz}$ comb was achieved. Moreover, pumping the resonator with $300 \mathrm{~W}$ power leads to an efficient comb broadening to a span over $250 \mathrm{~nm}$. The observation of a soliton step in AlGaAsOI platform has also been reported. This demonstration opens up many opportunities for ultra-efficient nonlinear photonics in this platform. Furthermore, it paves the way for fully integrated nonlinear PICs in the future.

This work is supported by a DARPA MTO DODOS contract (HR0011-15-C-055). We thank Gordon Keeler for fruitful discussions.

\section{References}

[1] D. T. Spencer et al., "An optical-frequency synthesizer using integrated photonics," Nature, vol. 557, no. 7703, pp. 8185,2018

[2] T. J. Kippenberg, A. L. Gaeta, M. Lipson, and M. L. Gorodetsky, "Dissipative Kerr solitons in optical microresonators.," Science, vol. 361, no. 6402, p. eaan8083, Aug. 2018.

[3] L. Chang et al., "Heterogeneously integrated GaAs waveguides on insulator for efficient frequency conversion," Laser Photon. Rev., 2018.

[4] M. Pu, L. Ottaviano, E. Semenova, and K. Yvind, "Efficient frequency comb generation in AlGaAs-on-insulator," Optica, vol. 3, no. 8, pp. 823-826, 2016.

[5] L. Chang et al., "Strong frequency conversion in heterogeneously integrated GaAs resonators," APL Photonics, vol. 4, no. 3, p. 036103, Mar. 2019.

[6] L. Chang et al., "Ultra-efficient frequency comb generation in AlGaAs-on-insulator microresonators," Sep. 2019. 